\title{
SOLVING DETERMINISTIC AND STOCHASTIC EQUILIBRIUM PROBLEMS VIA AUGMENTED WALRASIAN
}

\author{
Julio Deride \\ Mathematics \\ Univ. California, Davis \\ jderide@math.ucdavis.edu
}

\author{
Alejandro Jofré \\ CMM \& DIM \\ Universidad de Chile \\ ajofre@dim.uchile.cl
}

\author{
Roger J-B Wets \\ Mathematics \\ Univ. California, Davis \\ rjbwets@ucdavis.edu
}

\begin{abstract}
We described a method to solve deterministic and stochastic Walras equilibrium models based on associating with the given problem a bifunction whose maxinf-points turn out to be equilibrium points. The numerical procedure relies on an augmentation of this bifunction. Convergence of the proposed procedure is proved by relying on the relevant lopsided convergence. In the dynamic versions of our models, deterministic and stochastic, we are mostly concerned with models that equip the agents with a mechanism to transfer goods from one time period to the next, possibly simply savings, but also allows for the transformation of goods via production.
\end{abstract}

Keywords: Walras equilibrium, stochastic equilibrium, lopsided convergence, epi-convergence, augmented Walrasian, progressive hedging algorithm.

JEL Classification: C680, D580, C620

Date: $\quad$ May 29, 2022 


\section{Introduction}

The economic equilibrium model proposed by Arrow and Debreu [1] for a competitive economy implicitly assumes that the entire economic activity will take place in a single time span, implicitly instantly. As soon as one includes the agent's concerns about the future, one has to consider a dynamic component and take into account the uncertainty about this future. Of course, that's bound to enrich the model, raising in the process a wide variety of modeling issues. In this article we are only going to be concerned with numerical procedures to solve trimmed down stochastic Walras equilibrium models where goods are transferred from time 0 to time 1 via "home production" which includes the possibility of simple retention; the extension of this approach to include financial markets is presently under development.

The overall approach to the stochastic case is based on a result that allows us to proceed with the calculation of the equilibrium for each particular state, a decomposition type approach. This means that our first task is going to be the development of a method that will arrive at an equilibrium rather efficiently in a deterministic, but dynamic, environment. We start with the pure exchange model of Arrow-Debreu, next consider a two time-periods dynamic version and then proceed to deal with the stochastic version of this model. We rely on an augmentation method applied to what we call the Walrasian, essentially, the function 'supposedly solved' by the Walrasian auctioneer and a maxmin characterization of an equilibrium point. The fact that the theory allows us to proceed, in the iterative process, with approximate equilibria turns out to be critical in the development of the overall numerical scheme.

Our approach deviates, even in the deterministic case, from the path-breaking methods suggested by Scarf and Hansen [22], Eaves [8, 5], Saigal [21], and other approximation strategies described in the books by Judd [15], and Brown and Kubler [6]. These earlier methods are not efficient when the economies have a large number of goods or agents, even for reaching approximate equilibria. Moreover, in stochastic environments these results are prohibitively time-expensive.

In this paper we develop an approach based on an augmented Walrasian technique and a lopsided convergence approximation procedure, which allows us to cope with large equilibrium problems including uncertainty and heterogeneity on the agents. By using this approach we have designed a two-phase algorithm without computing derivatives of the demand function. We report several numerical experiments for equilibrium problems involving up to 5 agents, 7 goods and 10 stochastic scenarios, which can be easily expanded in number of agents and goods. Finally, the procedure proposed in this paper might be parallelized in terms of agents and the multi-start strategy.

\section{The Arrow-Debreu model}

To set the stage and fix terminology and notation, let's start with the barter, or pure exchange model, of Arrow-Debreu [7]. A finite number of (individual) agents $i \in \mathcal{I}$ with initial endowments $\left\{e_{i} \in \mathbb{R}^{L}, i \in \mathcal{I}\right\}$, consisting of a finite number of goods, to be bartered so as to maximize, individually, their upper semicontinuous (usc) concave utility functions $\left\{u_{i}: \mathbb{R}^{L} \rightarrow[-\infty, \infty), i \in \mathcal{I}\right\}$ that depend on the level of the acquisitions $x_{i}(p) \in \mathbb{R}^{L}$ of these goods, potentially for "consumption"; one refers to $X_{i}=\operatorname{dom} u_{i}=\left\{x \in \mathbb{R}^{L} \mid u_{i}(x)>-\infty\right\}$ as the survival sets; note that the 
concavity of $u_{i}$ implies that the survival set $X_{i}$ is convex, typically unbounded. The value to assign to each good, in this trading process, depends on a market price system $0 \neq p \in \mathbb{R}_{+}^{L}$ that will restrict each agent to limit the "market value" of its acquisitions to the "market value" of its endowment, i.e., $\langle p, x\rangle \leq\left\langle p, e_{i}\right\rangle$; since these prices don't necessarily reflect monetary prices, the "values" are often referred to as units of account. Given $p \in \mathbb{R}_{+}^{L}$, each agent maximizes its utility subject to its budgetary constraint, i.e.,

$$
x_{i}(p) \in \operatorname{argmax}_{x \in X_{i} \subset R_{+}^{L}} u_{i}(x)
$$

For the market to be in equilibrium the total demand must not exceed total supply, i.e., with $s$ designating the excess supply function,

$$
s(p)=\sum_{i \in \mathcal{I}}\left(e_{i}-x_{i}(p)\right) \geq 0 .
$$

Since, we haven't ruled out the possibility that at equilibrium the prices of some goods might turn out to be 0 , one can also write this condition in terms of a geometric variational inequality:

$$
-\sum_{i \in \mathcal{I}}\left(e_{i}-x_{i}(p)\right)=-s(p) \in N_{R_{+}^{L}}(p)
$$

where $N_{C}(p)$ denotes the normal cone of variational analyis [20] to the set $C$ at $p$, or still, must be solutions of the linear complementarity problem,

$$
0 \leq p \perp \sum_{i \in \mathcal{I}}\left(e_{i}-d_{i}(p)\right) \geq 0
$$

Since the budgetary constraints are positively homogeneous in $p$ and $p \neq 0$, no additional restriction is introduced by insisting that the price system should be scaled so that it lies in the unit simplex $\Delta^{L}=\left\{p \in \mathbb{R}_{+}^{L} \mid\langle p, e\rangle=1\right\}$. This is often included in the formulation of the problem to enable appealing to a fixed point argument to establish existence or to provide boundedness in the design of a computational scheme.

Additionally, we introduce a natural bound for each agent demand function $x_{i}(p) \leq \sum_{i \in \mathcal{I}} e_{i}$ (see $[7$, Ch.5]), i.e., no agent can demand more quantity of each good than the total amount available in the economy, which in turn obtains a bound for $s(p)>-\infty$.

\section{Augmented Walrasian}

Our assumptions, and notation introduced in the previous section, follow those of the article "Continuity properties of Walras equilibrium points" [12] which introduced the Walrasian function associated with this problem

$$
W(p, q)=\langle q, s(p)\rangle \text { on } \Delta \times \Delta
$$

where $p, q \in$ the (unit) price simplex $\Delta \subset \mathbb{R}^{n}$, and $s$ is our excess supply function as defined in the previous section. Moreover, the following lemma provides that every max-inf point of $W$ is an equilibrium price, i.e., if $\inf _{q \in \Delta} W(\bar{p}, q)=\sup _{p \in \Delta} \inf _{q \in \Delta} W(p, q)$ then $s(\bar{p}) \geq 0[12]$. 
3.1 Lemma (Walras equilibrium prices and maxinf-points). Every maxinf-point $\bar{p} \in \Delta$ of the Walrasian function $W$ such that $W(\bar{p}, \cdot) \geq 0$ on $\Delta$ is an equilibrium point.

Proof. If $\bar{p}$ is a maxinf-point of the Walrasian with $W(\bar{p}, \cdot) \geq 0$, it follows that for all unit vectors $e^{j}=(0, \ldots, 1, \ldots)$, the $j$-th entry is $1,\left\langle e^{j}, s(\bar{p})\right\rangle \geq 0$ which implies $s(\bar{p}) \geq 0$.

The condition of $W(\bar{p}, \cdot) \geq 0$ follows by the definition of $W$ and noting that for every price $p \in \Delta$, and under local nonsatiation preferences assumption, $W(p, p)=0$. Furthermore, the converse of lemma (3.1) also holds, i.e., every equilibrium point is a maxinf-point of $W$ [14, Prop.2.4].

Existence of equilibrium prices can be seen as the existence of max-inf points for the corresoponding Walrasian. Under general conditions for the upper-semicontinuity of the excess supply function, it is easy to see that $W$ is a Ky Fan function [2], and the existence of max-inf point is provided by [2, Theorem 6.3.5]

Since our basic approach, first suggested by A. Bagh [3], is related to that for the augmented Lagrangian, it's informative to consider the bifunction that might have led to the Walrasian in a standard non-convex duality scheme $[20, \S 11 . \mathrm{K}]$. Let's introduce a pre-Walrasian obtained as a restricted-partial conjugate, with respect to the $q$-variable, i.e., for all $p \in \Delta$

$$
V(p, u)=\sup _{z \in \Delta}[\langle u, z\rangle-W(p, z)] .
$$

$V(p, \cdot)$ is clearly convex and one can think of the family of bifunctions $\left\{V(\cdot, u), u \in \mathbb{R}^{n}\right\}$ as 'perturbations' of a 'fundamental' primal-problem

$$
\text { find } \hat{p} \in \operatorname{argmax}_{p \in \Delta} v(p) \quad \text { where } \quad v(p)=V(p, 0)=-\inf _{q \in \Delta}[W(p, q)] .
$$

By conjugacy, since the functions $q \mapsto W(p, q)$ on $\Delta$ are proper, lower semicontinuous (lsc) and convex, so are the functions $u \mapsto V(p, u)$. Note that $\min _{q \in \Delta}\langle q, s(p)\rangle$ will yield the $q$ that generates the smallest convex combination of the elements of $s(p)$. So, if for any $l, s_{l}(p)<0$, it follows that $v(p)>0$. Thus, $\hat{p}$ will be such that an element of the vector $s(\hat{p})$ will be as negative as possible it will minimize the $\ell^{\infty}$-norm of $s(p)$.

The process of going from $v$ to the collection $\left\{V(\cdot, u), u \in \mathbb{R}^{n}\right\}$ is well-understood; it can be viewed as associating to a particular optimization problem, $\max \{v(p) \mid p \in \Delta\}$, a perturbed collections that leads to the analysis of stability. But in our setting what is this particular optimization problem? It can be viewed as the Walrasian auctioneer's problem. It's easy to see that it's optimal value is 0 which is attained when the Walrasian auctioneer has selected a price system that yields an equilibrium. Generally it's not a concave function, and certainly not a strictly concave function, and thus one can't expect a unique maximizer which, precisely, correspond to the well-know fact that, in general, Walras equilibrium points are not unique.

In order to compute equilibrium points for an economy, we propose a strategy to find a max-inf point of $W$ by an approximating scheme. Our first goal is to build a family of approximating bifunctions by relying on an augmentation technique. Let $\sigma: \mathbb{R}^{n} \rightarrow \bar{R}$ be an augmenting function, i.e., it's convex, $\operatorname{argmin} \sigma=\{0\}$ and $\min \sigma=0$. Typically, $\sigma=|\cdot|$ is chosen to be a norm 
but depending on the application it could be quite different; recall that we can even choose $\sigma$ to take on the value $\infty$, for example, it could be a norm of some type restricted to a ball centered at 0 , or even more exotic.

Given the augmenting function $\sigma$ and a scalar $r>0$, the augmented Walrasian, by definition, is

$$
\tilde{W}_{r}(p, q)=\sup _{u \in R^{n}}\{\langle q, u\rangle-V(p, u)-r \sigma(u)\} .
$$

For a fixed $p$, considering the convexity of $V(p, \cdot)$ and $\sigma$, one can re-write the definition of $\tilde{W}_{r}$ as a partial conjugate w.r.t the $u$-variable. Additionally, by the property of conjugation of a sum and the definition of the epi-sum (\#), we have the following chain of identities

$$
\begin{aligned}
\tilde{W}_{r}(p, q) & =(V(p, \cdot)+r \sigma)^{*}(q) \\
& =\operatorname{cl}\left\{\left(\left(V(p, \cdot)^{*} \#(r \sigma)^{*}\right)(q)\right\}\right. \\
& =\inf _{z}\left\{(V(p, \cdot))^{*}(q-z)+(r \sigma)^{*}(q)\right\} \\
& =\inf _{z}\left\{W(p, q-z)+r \sigma^{*}\left(r^{-1} z\right)\right\}
\end{aligned}
$$

where $\sigma^{*}$ is the conjugate of $\sigma$, i.e., $\sigma^{*}(v)=\sup _{x}\{\langle v, x\rangle-\sigma(x)\}$. Thus, the augmented Walrasian function in its final form is the infimum of a convex function, and depending of our choice of $\sigma$, possibly quadratic or linear.

Additionally, we establish a definition for an approximating equilibrium point, given by a price such that the associated excess supply function is close to satisfy the market clearing condition. More precisely, the definition can be stated as follows:

3.2 Definition (approximate maxinf-points). For $\varepsilon \geq 0, p_{\varepsilon}$ is said to be an approximate equilibrium point or approximate maxinf-point of $W$ if $\inf W\left(p_{\varepsilon}, \cdot\right) \geq \sup \inf W-\varepsilon$, and the set of all such approximating maxinf-points is denoted by $\varepsilon$-argmaxinf $W$.

Note that given an approximating equilibrium price, $p_{\varepsilon}$, one can adjust the agents's intial endowments by a fraction of $\varepsilon$ and make $p_{\varepsilon}$ and equilibrium price.

The next step is to establish a connection between the convergence of augmented Walrasian approximating equilibrium points and the goal of finding an equilibrium price for the original economy. Considering the family of augmented Walrasian perturbations, and a sequence of their corresponding approximate max-inf points, one should be able to guarantee a convergence result of this sequence of points, given the convergence of the family of augmented functions. This condition can be obtained by appealing to lopsided convergence, or lop-convergence, of the augmented Walrasian to the Walrasian. Given the compactness of the domain, one doesn't have to appeal to the (more comprehensive) definition of lopsided convergence it suffices to refer to a more restrictive version, namely tight lopsided convergence; for the general definition and further details, consult $[13,14]$.

3.3 Definition (tight lopsided convergence). A sequence in finite-valued bivariate functions, $f_{V-b i v}\left(\mathbb{R}^{n+m}\right)$, defined over a compact set $C \times D,\left\{F^{\nu}: C \times D \rightarrow \mathbb{R}\right\}_{\nu \in N}$ lop-converges tightly to a function $F: C \times D \rightarrow \mathbb{R}$, also in $f_{V-b i v}\left(\mathbb{R}^{n+m}\right)$, if 
(a) for all $y \in D$, and all $\left(x^{\nu} \in C\right) \rightarrow x \in C$, there exists $\left(y^{\nu} \in D\right) \rightarrow y$ such that

$$
\limsup _{\nu} F^{\nu}\left(x^{\nu}, y^{\nu}\right) \leq F(x, y)
$$

(b) for all $x \in C$, there exists $\left(x^{\nu} \in C\right) \rightarrow x$ such that given any $\left(y^{\nu} \in D\right) \rightarrow y \in D$,

$$
\liminf _{\nu} F^{\nu}\left(x^{\nu}, y^{\nu}\right) \geq F(x, y)
$$

The desired convergence result for the equilibrium points follows from adaptating the tight lopconvergence given by [14, Theorem 3.2], to our case with compact (and invariant) domains.

3.4 Theorem (convergence of maxinf-points, [14, Theorem 3.2]). Let $C \times D$ be a compact subset of $\mathbb{R}^{n+m}$. When the bifunctions $\left\{F^{\nu}\right\}_{\nu \in N}$ lop-converge tightly to $F$, all in $f_{V}$-biv $(C \times D)$ with supinf $F$ finite, and $\varepsilon^{\nu} \searrow \varepsilon \geq 0$, then every cluster point $\bar{x} \in C$ of a sequence of $\varepsilon^{\nu}$-maxinf points of the bifunctions $F^{\nu}$ is an $\varepsilon$-maxinf point of the limit function $F$.

In particular, this implies that in these circumstances, every cluster point of a sequence of maxinf-points of the bifunctions $F^{\nu}$ is a maxinf-point of the lop-limit function $F$.

In order to obtain our convergence result for approximating maxinf points, the following result is an application of the previous theorem in our framework. It tell us that tight lopsided convergence of the augmented Walrasian entails convergence of equilibrium points.

3.5 Theorem (convergence of $\varepsilon$-maxinf points). Suppose that $p \mapsto s(p)$ is usc on $\Delta$. Consider the non-negative sequences $\left\{r^{\nu}, \nu \in \mathbb{N}\right\}$ and $\left\{\varepsilon^{\nu}, \nu \in \mathbb{N}\right\}$ such that $r^{\nu} \nearrow \infty, \varepsilon^{\nu} \searrow \varepsilon \geq 0$. Let $\left\{W^{\nu}, \nu \in \mathbb{N}\right\}$ be a family of augmented Walrasian functions associated which each augmenting parameter $r^{\nu}$. Let $p^{\nu} \in \varepsilon^{\nu}$-argmaxinf $W^{\nu}$ and $\bar{p}$ be any cluster point of $\left\{p^{\nu}, n u \in \mathbb{N}\right\}$. Then $\bar{p} \in \varepsilon$-argmaxinf $W$.

Proof. It suffices to show that $\left\{W^{\nu}, \nu \in \mathbb{N}\right\}$ lop-converges tightly to $W$ and conclude by Theorem (3.4) the convergence of a (sub)sequence of $\varepsilon^{\nu}$-maxinf points. In order to prove tight lopsided convergence, let $q \in \Delta,\left\{p^{\nu}, \nu \in \mathbb{N}\right\} \rightarrow p \in \Delta$. Define $q^{\nu} \equiv q, \nu \in \mathbb{N}$. Then

$$
W^{\nu}\left(p^{\nu}, q^{\nu}\right)=\inf _{z \in R^{n}}\left\{W\left(p^{\nu}, z\right)+r^{\nu} * \sigma^{*}\left(q^{\nu}-z\right)\right\} \leq W\left(p^{\nu}, q^{\nu}\right),
$$

and as the function $p \mapsto s(p)$ is usc,

$$
\limsup W^{\nu}\left(p^{\nu}, q^{\nu}\right) \leq \limsup W\left(p^{\nu}, q\right) \leq W(p, q) .
$$

On the other hand, let $p \in \Delta$ and $\left\{q^{\nu}, \nu \in \mathbb{N}\right\} \rightarrow q$. By compactness of $\Delta, q \in \Delta$ and defining $p^{\nu}=p, \nu \in \mathbb{N}, W^{\nu}(p, q)$ is the inf-projection of the function $F^{\nu}(q, z)=W(p, q-z)+r^{\nu} * \sigma^{*}(z)$ in the $z$-variable. Thus, $F^{\nu}$ is level bounded in $z$ locally uniform in $q$ and therefore $W^{\nu}(p, \cdot)$ is lsc by [20, Theorem 1.17]. Finally,

$$
\liminf W^{\nu}\left(p^{\nu}, q^{\nu}\right) \geq W(p, q),
$$

since for any $q_{0} \in \Delta, W^{\nu}\left(p, q_{0}\right) \rightarrow W\left(p, q_{0}\right)$ as $\nu \rightarrow \infty$ and the conclusion follows from a standard diagonal argument.

The following inmediate corollary of this theorem, with $\varepsilon=0$, plays a pivotal role form a numerical viewpoint 
3.6 Corollary ( $\varepsilon$-maxinf and equilibrium points). Let $\varepsilon^{\nu} \searrow 0$. Then, every cluster point of a sequence of $\varepsilon^{\nu}$-approximating equilibrium points of a sequence of augmented Walrasian functions is an equilibrium point for the original economy.

The major thrust of the eventual algorithmic procedures is to replace finding local near maxinfpoints of $W$ by finding a local saddle point of a $\tilde{W}^{\nu}$ for $\nu$ large enough (but not too large to avoid numerical instabilities). Under this scheme, there are several options for choosing the augmenting function $\sigma$. For example, one can consider $\sigma=|\cdot|$ a norm whose dual norm will be denoted by $|\cdot|_{o}$, then one can express the augmented Walrasian as

$$
\tilde{W}^{\nu}(p, q)=\min _{z}\left[W(p, z) \mid z \in \mathbb{B}_{0}\left(q, r_{\nu}\right) \cap \Delta\right],
$$

where $\mathbb{B}_{0}\left(q, r_{\nu}\right)$ is the dual ball with center in $q$ and radius $r_{\nu}$. Alternatively, for $\sigma$ be the self-dual function, i.e., $\sigma=\frac{1}{2}|\cdot|{ }_{2}^{2}$, the augmented Walrasian takes the form

$$
\tilde{W}^{\nu}(p, q)=\min _{z}\left[W(p, z)+\frac{1}{2 r^{\nu}}|z-q|_{2}^{2} \mid z \in \Delta\right] .
$$

There is quite a variety of procedures for finding these near local saddle-points. One possible procedure to solve the problem at hand is described next:

- At iteration $\nu+1$, given $\left(p^{\nu}, q^{\nu}\right)$ with $r=r_{\nu+1}\left(\geq r_{\nu}\right)$, the Phase I (or primal) consists in solving

$$
q^{\nu+1} \in \operatorname{argmin}_{q \in \Delta} \tilde{W}^{\nu+1}\left(p^{\nu}, q\right)
$$

note that the 'internal' minimization is either that of a linear form on a ball, this seems to favor $|\cdot|_{o}$ as the $\ell^{\infty}$-norm, or the self-dual augmenting function which yields an immediate solution.

- How to carry out the next step will depend on the 'shape' and the properties of the demand functions. For example, this turns out to be rather simple when the utility functions are of the Cobb-Douglas type, defining the Phase II (or dual) as finding

$$
p^{\nu+1} \in \operatorname{argmax}_{p \in \Delta} \tilde{W}^{\nu+1}\left(p, q^{\nu+1}\right)
$$

In virtue of the corollary (3.6), we know that as $r_{\nu} \nearrow \infty, p^{\nu} \rightarrow \bar{p}$ a maxinf-point of $W$, equivalently an equilibrium price system for Walras' problem. The strategy for increasing $r_{\nu}$ should take into account (i) numerical stability, i.e., keep $r_{\nu}$ as small as possible and (ii) efficiency, i.e., increase $r_{\nu}$ sufficiently fast to guarantee accelerated convergence.

\subsection{Numerical implementation for the Arrow-Debreu model.}

The proposed algorithm was implemented in Pyomo (Python Optimization Modeling Objects, [10]), a mathematical programming language based on Python. The problems that we solve come with the following features: 
- In order to describe the economy, we consider utility functions of Cobb-Douglas and Constant Elasticity of Substitution (CES) type, and strictly positive aggregated initial endowment for every good.

- For the selection of the augmenting function $\sigma$, we primarily considered the self-dual type, given by $\sigma=\frac{1}{2}|\cdot|_{2}^{2}$.

- For the agent problem, everyone has to maximize a concave utility function over a linear constrained set determined by budgetary constraint and nonegativity of the solution. This problem is solved using the interior point method, Ipopt, implemented by [23] (which gives satisfactory results for problems of this nature).

- Phase I consists of the minimization of a quadratic objective function over the simplex of prices. This is solved using Gurobi solver [9], a state-of-the-art and efficient algorithm.

- Phase II is the critical step of the entire augmented Walrasian algorithmic framework. We need to overcome the (typical) lack of concavity of the objective function. Thus, the maximization is done without considering first order information and relying on BOBYQA algorithm [18]. which performs a sequentially local quadratic fit of the objective functions, over box constraints, and solves it using a trust-region method. All the examples were run on a $3.30 \mathrm{GHz}$ Intel Core i3-3220 processor with $4 \mathrm{~GB}$ of RAM memory, under Ubuntu 12.04 operating system.

In what follows, a set of numerical examples is described. The first example corresponds to a toy model, wich turns out to be useful in the general description of how the algorithm acts in every interation to get to an equilibrium price. The second one provides a direct benchmark for the performance between our algorithm and a classical example in the literature, provided by Scarf [16, Chapter 4]. This section ends with a larger example of an exchange economy (with symmetric agents), reflecting the computational power of the augmented Walrasian approach.

3.7 Example (symmetric agents). To test the overall performance of the algorithm we start with a basic example. Consider an economy of three goods and two agents, with utility functions within the CES family, i.e.,

$$
u_{i}(x)=\left(\sum_{j=1}^{3}\left(a_{i, j}\right)^{\frac{1}{b_{i}}}\left(x_{j}\right)^{\frac{b_{i}-1}{b_{i}}}\right)^{\frac{b_{i}}{b_{i}-1}}
$$

with survival sets $X_{i}=\left[10^{-3}, \infty\right)^{2}$, for each agent. In this first example, the agents are symmetric, i.e., their utility functions' coefficients are equal, given by $a_{i, j}=\frac{1}{3}, i=1,2, j=1,2,3$ and $b_{i}=\frac{1}{2}, i=1,2$, as well as their initial endowments $e_{i, j}=1, j=1,2,3, i=1,2$. It is easy to see that, by symmetry of the agents, the equilibrium price for this economy is given by $p^{*}=\left(\frac{1}{3}, \frac{1}{3}, \frac{1}{3}\right)$, and it is unique. Computationally, we initialize the algorithm at an arbitrary point of the simplex, in this case, $p^{0}=(0.12,0.56,0.32)$. The trajectory of prices $\left\{p^{\nu}\right\}$ and excess supply evaluations $s\left(p^{\nu}\right)$ performed by our algorithm are depicted in Figure (1). The first graph describes the price evolution, where each good is represented by a line (prices are scaled 
Table 1: Initial endowments for Example 3.8

\begin{tabular}{crrrrrrrrrr}
\hline Consumer & \multicolumn{10}{c}{ Initial endowments $e_{i j}$} \\
\hline 1 & 0.6 & 0.2 & 0.2 & 20.0 & 0.1 & 2.0 & 9.0 & 5.0 & 5.0 & 15.0 \\
2 & 0.2 & 11.0 & 12.0 & 13.0 & 14.0 & 15.0 & 16.0 & 5.0 & 5.0 & 9.0 \\
3 & 0.4 & 9.0 & 8.0 & 7.0 & 6.0 & 5.0 & 4.0 & 5.0 & 7.0 & 12.0 \\
4 & 1.0 & 5.0 & 5.0 & 5.0 & 5.0 & 5.0 & 5.0 & 8.0 & 3.0 & 17.0 \\
5 & 8.0 & 1.0 & 22.0 & 10.0 & 0.3 & 0.9 & 5.1 & 0.1 & 6.2 & 11.0 \\
\hline
\end{tabular}

by a factor of 100). The second graph depicts the behaviour of the corresponding excess supply function, where each good is again represented by a line. The adjustment process of the prices shows the Walrasian auctioneer's problem, where in every iteration, the algorithm identifies the good of the excess supply function with the least value, and performs an iteration adjusting its price for the next period. As the algorithm progresses, it converges to the equilibrium price.

Figure 1: Homogeneus Agents (Example 3.7)

3.8 Example (exchange economy; Scarf example). Consider the example described in H. Scarf in [16, Chapter 4]: exchange economy involving five type of consumers and ten comodities. The initial endowment for each agent is given by Table 3.8.

The utility functions correspond to the CES-type, for which the parameters $a_{i j}$ and $b_{i}$ for each consumer are described in Table 3.8.

Table 2: Utility parameters for Example 3.8

\begin{tabular}{crrrrrrrrrrr}
\hline Consumer & \multicolumn{10}{c}{ Utility parameters } \\
& \multicolumn{10}{c}{$a_{i, j}$} \\
\hline 1 & 1.0 & 1.0 & 3.0 & 0.1 & 0.1 & 1.2 & 2.0 & 1.0 & 1.0 & 0.07 & 2.0 \\
2 & 1.0 & 1.0 & 1.0 & 1.0 & 1.0 & 1.0 & 1.0 & 1.0 & 1.0 & 1.0 & 1.3 \\
3 & 9.9 & 0.1 & 5.0 & 0.2 & 6.0 & 0.2 & 8.0 & 1.0 & 1.0 & 0.2 & 3.0 \\
4 & 1.0 & 2.0 & 3.0 & 4.0 & 5.0 & 6.0 & 7.0 & 8.0 & 9.0 & 10.0 & 0.2 \\
5 & 1.0 & 13.0 & 11.0 & 9.0 & 4.0 & 0.9 & 8.0 & 1.0 & 2.0 & 10.0 & 0.6 \\
\hline
\end{tabular}

The algorithm was set with the self-dual augmenting function, and the centroid of the simplex as the initial point. Additionally, the augmenting parameter is updated by $r^{\nu}=1.259$. The trajectory of prices $\left\{p^{\nu}\right\}$ and the corresponding sequence of excess supply evaluations are depicted in Figure 2. In this example, the convergence to an approximate equilibrium point for $\varepsilon=10^{-1}$ 
is obtained within 37 iterations, taking a machine time of 114 [min]; for $\varepsilon=10^{-2}$, 53 iterations were required taking 179 [min]. The price is given by

$$
p^{*}=(18.4,11.0,9.9,4.4,12.5,7.7,11.7,10.2,9.9,4.3)
$$

As in the previous example, the price sequence describes a trajectory that can be associated with the Walrasian auctioneer's problem. Similar results are obtained with different starting points, as well as different augmenting sequences.

Figure 2: Scarf's example (Example 3.8)

3.9 Example (large scale, symmetric agents economy). In this example, we consider a larger economy, with a total of 50 consumption goods and 10 agents with homogeneous CES utility functions defined over survival sets given by $\left[10^{-3}, \infty\right)^{50}$. The starting price is a random point in the simplex. As expected, the trajectory of the approximating prices $\left\{p^{\nu}\right\}$ converges to the unique equilibrium price system, in which every good has the same price, i.e., $p_{g}=\frac{1}{50}, g=1, \ldots, 50$. The converge of the sequence of prices, $\left\{p^{\nu}\right\}$ and the corresponding sequence of excess suppy functions $\left\{s\left(p^{\nu}\right)\right\}$ is illustrated in Figure 3.

Figure 3: Large scale, symmetric agents (Example 3.9)

From the examples previously described, a crucial observation can be made regarding the stability of the iterative process: the algorithm approaches an approximating equilibrum with about half of the total iterations. This behaviour is robust in every simulation performed, and one find a reason in the introduction of the augmenting function.

It's noteworthy that, in all cases, after a few iteration, the procedure finds an approximate equilibrium which one should be able to exploit when dealing with equilibirum problems in a stochastic environment.

\section{Dynamic deterministic equilibrium model}

As a stepping stone to the solution of stochastic Walras equilibrium models, we are going to rely on solving, efficiently, deterministic dynamic versions of the Walras equilibrium model. Our starting point is a two-stage model that's formulated as follows: Given a price system $p=\left(p^{0}, p^{1}\right)$ 
with $p^{t}$ the price vector in vigor at time $t$, each agent $i \in \mathcal{I}$ determines its optimal consumption plan $\bar{x}=\left(\bar{x}_{i}^{0}, \bar{x}_{i}^{1}\right)$ as the solution of the following utility maximization problem,

$$
\begin{aligned}
\max _{x^{0}, y, x^{1}} & u_{i}^{0}\left(x^{0}\right)+u_{i}^{1}\left(x^{1}\right) \\
\text { so that } & \left\langle p^{0}, x^{0}+T_{i}^{0} y\right\rangle \leq\left\langle p^{0}, e_{i}^{0}\right\rangle, \\
& \left\langle p^{1}, x^{1}\right\rangle \leq\left\langle p^{1}, e_{i}^{1}+T_{i}^{1} y\right\rangle, \\
& x^{0} \in X_{i}^{0}, \quad y \in Y_{i}, \quad x^{1} \in X_{i}^{1},
\end{aligned}
$$

where $u_{i}^{t}, e_{i}^{t}$ and $X_{i}^{t}$ are the utility functions, the endowments and the survival sets for agent $i$ at time $t=0,1$. As in $\S 2$, the utility functions are assumed to be usc and concave, providing the convexity of the corresponding survivals sets. The vector $y$ determines a set of activities selected by agent $i$ at time 0 that requires an input of goods $T_{i}^{0} y$ and produces a deterministic output $T_{i}^{1} y$ at time 1 . The closed convex cone $Y_{i} \subset \mathbb{R}^{m}$ determines the set of potential activities that are at the disposal of agent- $i$; in many instances one would simply have $Y_{i}=\mathbb{R}_{+}^{m}$ but not necessarily in general. One can think of the pair of matrices $\left(T_{i}^{0}, T_{i}^{1}\right)$ as determining an input/output (home production) process that could simply be savings including enhancements or deterioration, or investment-activities, and so on. Of course, the agent chooses $y$ so as to maximize its overall utility; note, $u_{i}^{1}$ could include a discount factor that doesn't have to be made explicit here.

The excess supply function $s(p)=\left(s^{0}\left(p^{0}, p^{1}\right), s^{1}\left(p^{0}, p^{1}\right)\right)$ is given as usual as the difference between the total amount of goods available in each time period and the total endowments adjusted by the goods used or generated by the input/output process, i.e.,

$$
\begin{aligned}
& s^{0}(p)=\sum_{i \in \mathcal{I}}\left[e_{i}^{0}-\left(x_{i}^{0}(p)+T_{i}^{0} y_{i}(p)\right)\right], \\
& s^{1}(p)=\sum_{i \in \mathcal{I}}\left[\left(e_{i}^{1}+T_{i}^{1} y_{i}(p)\right)-x_{i}^{1}(p)\right],
\end{aligned}
$$

where $\left(x_{i}^{0}(p), y_{i}(p), x_{i}^{1}(p)\right)$ is the optimal solution for agent $i$ of its utility maximization problem.

The Walrasian, $W: \Delta^{2} \times \Delta^{2} \rightarrow \mathbb{R}$ is defined by

$$
W(p, q)=\langle q, s(p)\rangle=\left\langle\left(q^{0}, q^{1}\right),\left(s^{0}\left(p^{0}, p^{1}\right), s^{1}\left(p^{0}, p^{1}\right)\right)\right\rangle .
$$

$\bar{p}=\left(\bar{p}^{0}, \bar{p}^{1}\right)$ is and equilibrium price system if $s(\bar{p}) \geq 0$. As in the static (one-stage) model, it can be shown that such a $\bar{p}$ is a maxinf-point of the Walrasian and its existence is provided as $W$ is a Ky Fan function. One possible approach in finding such a maxinf-point is based on the Augmented Walrasian approach described in $§ 3$.

4.1 Theorem (dynamic deterministic maxinf-points). Consider the Walrasian function $W$ for the previous economy. Assuming local nonsatiation preferences, every maxinf-point $\bar{p}=\left(\bar{p}^{0}, \bar{p}^{1}\right)$ of $W$ is an equilibrium point, i.e., $s^{0}(\bar{p}) \geq 0$ and $s^{1}(\bar{p}) \geq 0$.

Proof. Adapting the same pattern of proof as in Lemma 3.1, for every price system $p=\left(p^{0}, p^{1}\right)$, $\left\langle p^{0}, s^{0}(p)\right\rangle=0$ and $\left\langle p^{1}, s^{1}(p)\right\rangle=0$. Then, if $\bar{p}$ is a maxinf-point of $W, W(\bar{p}, \cdot) \geq 0$, and it follows that for vectors $q=\left(e^{j}, \bar{p}^{1}\right)$ defined for every unit vector $e^{j}, 0 \leq\langle q, s(\bar{p})\rangle=\left\langle e^{j}, s^{0}(\bar{p})\right\rangle+\left\langle\bar{p}, s^{1}(\bar{p})\right\rangle$ which implies $s^{0}(\bar{p}) \geq 0$. Analogously, taking $q=\left(\bar{p}^{0}, e^{j}\right)$ it follows that $s^{1}(\bar{p}) \geq 0$. 
4.2 Theorem (convergence of $\varepsilon$-maxinf points and equilibrium). Suppose that $p \mapsto s(p)$ is usc on $\Delta$. Consider the non-negative sequences $\left\{r^{\nu}: \nu \in \mathbb{N}\right\}$ and $\left\{\varepsilon^{\nu}: \nu \in \mathbb{N}\right\}$ such that $r^{\nu} \nearrow \infty$, $\varepsilon^{\nu} \searrow \varepsilon \geq 0^{1}$. Let $\left\{W^{\nu}: \nu \in \mathbb{N}\right\}$ be a family of Augmented Walrasian functions associated which each augmenting parameter $r^{\nu}$. Let $p^{\nu} \in \varepsilon^{\nu}$-argmaxinf $W^{\nu}$ and $\bar{p}$ be a cluster point of $\left\{p^{\nu}: \nu \in \mathbb{N}\right\}$. Then $\bar{p} \in \varepsilon$-argmaxinf $W$. In particular, when $\varepsilon=0, \bar{p}$ is an equilibrium point.

Proof. The tight lop-convergence of the augmented Walrasian $\left\{W^{\nu}: \nu \in \mathbb{N}\right\}$ follows the same arguments as those cwin the proof of Theorem 3.5 and the conclusion follows from Theorem 3.4.

\subsection{Dynamic model: a solution strategy}

For a fixed choice of activities $y_{i}$, the two-stage deterministic model is essentially just an extension of a one-stage problem. With $y_{i}=\bar{y}_{i} \in Y_{i}$, after dropping reference to agent- $i$, the problem reads:

$$
\begin{aligned}
& \max _{\left(x^{0}, x^{1}\right)} u^{0}\left(x^{0}\right)+u^{1}\left(x^{1}\right) \\
& \text { so that }\left\langle p^{0}, x^{0}\right\rangle \leq\left\langle p^{0}, e^{0}-T^{0} \bar{y}\right\rangle, \\
&\left\langle p^{1}, x^{1}\right\rangle \leq\left\langle p^{1}, e^{1}+T^{1} \bar{y}\right\rangle, \\
& x^{0} \in X^{0}, \quad x^{1} \in X^{1},
\end{aligned}
$$

In fact, the problem is then separable, i.e., it can be solved by maximizing separately in the $x^{0}$ and $x^{1}$ variables:

$$
\begin{aligned}
& \max _{x^{0} \in X^{0}} u^{0}\left(x^{0}\right) \text { so that }\left\langle p^{0}, x^{0}\right\rangle \leq\left\langle p^{0}, e^{0}-T^{0} \bar{y}\right\rangle, \\
& \max _{x^{1} \in X^{1}} u^{1}\left(x^{1}\right) \text { such that }\left\langle p^{1}, x^{1}\right\rangle \leq\left\langle p^{1}, e^{1}+T^{1} \bar{y}\right\rangle .
\end{aligned}
$$

If these problems are of the Cobb-Douglas or CES-type, one can find (closed-form) explicit solutions to these problems at negligible computational cost.

The agent's problem can now be seen as finding the best $y \in Y$ that will maximize the overall rewards. With

$$
\begin{aligned}
r(y)= & \sup _{x^{0} \in X^{0}}\left\{u^{0}\left(x^{0}\right) \mid\left\langle p^{0}, x^{0}\right\rangle \leq\left\langle p^{0}, e^{0}-T^{0} y\right\rangle\right\} \\
& +\sup _{x^{1} \in X^{1}}\left\{u^{1}\left(x^{1}\right) \mid\left\langle p^{1}, x^{1}\right\rangle \leq\left\langle p^{1}, e^{1}+T^{1} y\right\rangle\right\},
\end{aligned}
$$

the agent's problem can be translated to:

$$
\text { find } y^{*} \in \operatorname{argmax}_{y \in Y} r(y) \text {. }
$$

We refer to this reduction as the transfer first approach and the algorithmic procedure to solve it (nonlinear convex optimization problem) very much depends on the properties of $r$. In the CobbDouglas or CES case, the function $r$ is twice differentiable and one can find explicit expressions

\footnotetext{
${ }^{1}$ Note that the equilibrium case $\varepsilon=0$ is included.
} 
for the gradient and the Hessian of $r$. When $Y=\mathbb{R}_{+}^{m}$, the problem boils down to maximizing a convex function on the non-negative orthant. Assuming further that $r$ is differentiable, the optimality conditions read:

$$
\text { for } k=1, \ldots, m, \quad y_{k}^{*} \geq 0, \quad \frac{\partial}{\partial y_{k}} r\left(y^{*}\right) \leq 0, \quad y_{k}^{*} \frac{\partial}{\partial y_{k}} r\left(y^{*}\right)=0 .
$$

A number of specialized algorithmic procedures have been designed for precisely this problemtype.

\subsection{The Cobb-Douglas case}

The utility function of agent- $i$ takes the form

$$
u_{i}(x)=\prod_{j=1}^{n} x_{j}^{\beta_{i, j}} \quad \text { with } \sum_{j=1} \beta_{i, j}=1, \quad \beta_{i, j} \geq 0 .
$$

For $p \in \Delta$ and assuming that the survival set $X_{i}=\mathbb{R}_{+}^{n}$, agent- $i$ solution is

$$
\text { for } j=1, \ldots, n, \quad \bar{x}_{i, j}(p)=\frac{\beta_{i, j}}{p_{j}} \sum_{l=1}^{n} p_{l} e_{i, l} ;
$$

the endowment of agent- $i: e_{i}=\left(e_{i, 1}, \ldots, e_{i, n}\right)$ and the utility attached to this solution:

$$
u_{i}\left(\bar{x}_{i}\right)=\alpha_{i}(p)\left(\sum_{l=1}^{n} p_{l} e_{i, l}\right) \quad \text { where } \alpha_{i}(p)=\prod_{j=1}^{N}\left(\frac{\beta_{i, j}}{p_{j}}\right)^{\beta_{i, j}} .
$$

For the dynamic model, once the activity levels $y \geq 0$ are fixed, the problem becomes separable (per-stage) and the solution takes the same form provided that $y$ is chosen so that $e_{i}^{0}-T_{i}^{0} y$ remains non-negative, otherwise agent- $i$ would enter the exchange market with a negative quantity of certain goods. It's implicitly assumed that the technology matrices $T_{i}^{0}, T_{i}^{1}$ are non-negative; negative entries in $T_{i}^{0}$ would imply goods-production at time 0 and negative entries in $T_{i}^{1}$ would imply negative outputs would be generated by certain technologies at time 1 . Hence, assuming that $T_{i}^{0} y \leq e_{i}^{0}$, the solutions (consumption vectors) that result from the choice of $y$ and $p=$ $\left(p^{0}, p^{1}\right) \in \Delta \times \Delta$ would be

$$
\text { for } j=1, \ldots, n, \quad \bar{x}_{i, j}^{0}\left(p^{0}\right)=\frac{\beta_{i, j}^{0}}{p_{j}^{0}} \sum_{l=1}^{n} p_{l}^{0}\left(e_{i, l}^{0}-\left\langle T_{i, l}^{0}, y\right\rangle\right) ;
$$

where $T_{i, l}^{0}$ is the $l$ th row of $T_{i}^{0}$,

$$
\text { for } j=1, \ldots, n, \quad \bar{x}_{i, j}^{1}\left(p^{1}\right)=\frac{\beta_{i, j}^{1}}{p_{j}^{1}} \sum_{l=1}^{n} p_{l}^{1}\left(e_{i, l}^{1}+\left\langle T_{i, l}^{1}, y\right\rangle\right) ;
$$

and consequently,

$$
\begin{aligned}
r_{i}(y) & =u_{i}^{0}\left(\bar{x}^{0}\right)+u_{i}^{1}\left(\bar{x}^{1}\right) \\
& =\alpha_{i}^{0}\left(p^{0}\right)\left(\sum_{l=1}^{n} p_{l}^{0}\left(e_{i, l}^{0}-\left\langle T_{i, l}^{0}, y\right\rangle\right)\right)+\alpha_{i}^{1}\left(p^{1}\right)\left(\sum_{l=1}^{n} p_{l}^{1}\left(e_{i, l}^{1}+\left\langle T_{i, l}^{1}, y\right\rangle\right)\right)
\end{aligned}
$$

As detailed in $\S 4.1$, the optimization problem for agent- $i$ is reduced to

$$
\text { find } \bar{y}_{i} \text { that maximizes } r_{i}(y) \text { such that } T_{i}^{0} y \leq e_{i}^{0}, y \in \mathbb{R}_{+}^{m} \text {. }
$$

This is a linear programming problem whose feasible region is bounded and non-empty; $y=0$ is always a feasible solution. 


\subsection{The Constant Elastiticity of Substitution case.}

If the utility functions for agent $i$ take the following form

$$
\begin{aligned}
& u_{i}^{0}\left(x^{0}\right)=\left(\sum_{j=1}^{n}\left(a_{i, j}^{0}\right)^{\frac{1}{b_{i}^{0}}}\left(x_{j}^{0}\right)^{\frac{b_{i}^{0}-1}{b_{i}^{0}}}\right)^{\frac{b_{i}^{0}}{b_{i}^{0}-1}} . \\
& u_{i}^{1}\left(x^{1}\right)=\left(\sum_{j=1}^{n}\left(a_{i, j}^{1}\right)^{\frac{1}{b_{i}^{1}}}\left(x_{j}^{1}\right)^{\frac{b_{i}^{1}-1}{b_{i}^{1}}}\right)^{\frac{b_{i}^{1}}{b_{i}^{1}-1}} .
\end{aligned}
$$

Then, the KKT optimality conditions ([20],) are satisfied if, and only if, the budget constraint is active. On the other hand, each agent must satisfy the constraint for feasibility $T_{i}^{0} y \leq e_{i}^{0}$. Then, for a given a feasible $y \in Y_{i}$, we can find an explicit solution, given by

$$
\text { for } j=1, \ldots, n, \quad \bar{x}_{i, j}^{0}(p)=\frac{a_{i, j}^{0}}{\left(p_{j}^{0}\right)^{b_{i}^{0}} \sum_{k=1}^{n}\left(p_{k}^{0}\right)^{1-b_{i}^{0} a_{i, k}^{0}}} \sum_{l=1}^{n} p_{l}^{0}\left(e_{i, l}^{0}-\left\langle T_{i, l}^{0}, y\right\rangle\right) \text {; }
$$

where $T_{i, l}^{0}$ is the $l$ th row of $T_{i}^{0}$,

$$
\text { for } j=1, \ldots, n, \quad \bar{x}_{i, j}^{1}\left(p^{1}\right)=\frac{a_{i, j}^{1}}{\left(p_{j}^{1}\right)^{b_{i}^{1}} \sum_{k=1}^{n}\left(p_{k}^{1}\right)^{1-b_{i}^{1}} a_{i, k}^{1}} \sum_{l=1}^{n} p_{k}^{1}\left(e_{i, l}^{1}+\left\langle T_{i, l}^{1}, y\right\rangle\right) ;
$$

Defining for agent- $i$

$$
\text { for } t=1,2, \quad \theta_{i}^{t}(p)=\left(\sum_{j=1}^{n}\left(a_{i, j}^{t}\right)^{\frac{1}{b_{i}^{t}}}\left(\frac{a_{i, j}^{t}}{\left(p_{j}^{t}\right)^{b_{i}^{t}}} \frac{1}{\sum_{k=1}^{n}\left(p_{k}^{t}\right)^{1-b_{i}^{t}} a_{i, k}^{t}}\right)^{\frac{b_{i}^{t}-1}{b_{i}^{t}}}\right)^{\frac{b_{i}^{t}}{b_{i}^{t}-1}} .
$$

consequently

$$
\begin{aligned}
r_{i}(y) & =u_{i}^{0}\left(\bar{x}^{0}\right)+u_{i}^{1}\left(\bar{x}^{1}\right) \\
& =\theta_{i}^{0}\left(p^{0}\right)\left(\sum_{l=1}^{n} p_{l}^{0}\left(e_{i, l}^{0}-\left\langle T_{i, l}^{0}, y\right\rangle\right)\right)+\theta_{i}^{1}\left(p^{1}\right)\left(\sum_{l=1}^{n} p_{l}^{1}\left(e_{i, l}^{1}+\left\langle T_{i, l}^{1}, y\right\rangle\right)\right)
\end{aligned}
$$

This is a linear function of $y$. Thus, if $Y_{i}=\mathbb{R}_{+}^{m}$, the problem for each agent is given by find $\bar{y}_{i}$ that maximizes $r_{i}(y)$ such that $T_{i}^{0} y \leq e_{i}^{0}, y \in \mathbb{R}_{+}^{m}$. 


\section{Stochastic Equilibrium.}

\subsection{The agent's problem.}

In an uncertain (stochastic) environment the agent's problem two-stage problem can be formulated as follows:

$$
\begin{aligned}
\max _{x^{0}, y, x^{1}} & u_{i}^{0}\left(x^{0}\right)+E_{i}\left\{u_{i}^{1}\left(\boldsymbol{\xi}, x_{\boldsymbol{\xi}}^{1}\right)\right\} \\
\text { So that } & \left\langle p^{0}, x^{0}+T_{i}^{0} y\right\rangle \leq\left\langle p^{0}, e_{i}^{0}\right\rangle, \\
& \left\langle p_{\xi}^{1}, x_{\xi}^{1}\right\rangle \leq\left\langle p_{\xi}^{1}, e_{i, \xi}^{1}+T_{i, \xi}^{1} y\right\rangle, \quad \forall \xi \in \Xi \\
& y \in \mathbb{R}_{+}^{m}, \quad x^{0} \in X_{i}^{0}, \quad x_{\xi}^{1} \in X_{i . \xi}^{1}, \quad \forall \xi \in \Xi,
\end{aligned}
$$

where, the utility functions are usc, concave and the survival sets are convex and unbounded. Additionally, the set $\Xi$ consists of a finite number of possible states (scenarios) and $E_{i}\{\cdot\}$ indicates that agent- $i$ is calculating the expectation with respect to agent- $i$ beliefs, i.e., to each possible state $\xi \in \Xi$, agent- $i$ assigns a probability $\pi_{i, \xi} \geq 0$ such that $\sum_{\xi \in \Xi} \pi_{i, \xi}=1$. It's possible, although unlikely, that all agents have the same information about the future in which case these probabilities wouldn't depend on $i$. As before, the agents set up their trades in full knowledge of the suggested price system, eventually an equilibrium price system,

$$
p=\left(p^{0},\left(p_{\xi}^{1}\right)_{\xi \in \Xi)}\right)
$$

in particular, $p_{\xi}^{1}$ is known for every contingency $\xi \in \Xi$. Note that the goods required $T_{i}^{0} y$ to carry out activities at level $y$ are still well determined, the output at time 1 is now stochastic, namely $T_{i, \xi}^{1} y$. This reflects a more realistic view of the output process. Even in the simple case of savings via buying certificates of deposit, bonds or stocks, their value at time 1 can't be known with certainty. This is even more so, if the activities are decisions involving manufacturing, the marketing or distribution of goods (perishable or not) and so on.

The agents' problems are thus (two-stage) stochastic programs with recourse [4] with stochastic entries in the right-hand side $\left\langle p_{\xi}^{1}, e_{i, \xi}^{1}\right\rangle$, the so-called technology matrix $T_{i, \xi}^{\top}$ and the recourse matrix $p_{\xi}^{1}$; the recourse decisions are $x_{i, \xi}^{1}$. Under the 'usual' conditions that guarantee the existence of an equilibirum price system, recalled in $[12,17]$, these stochastic programs are necessarily feasible; note however that straightforward feasibility of these stochastic programs doesn't really require such stringent conditions, for example, one could rely on an adaptation of the ample survivavibility assumption introduced in [11]. From the stochastic programming viewpoint these conditions can be viewed as sufficient conditions to guarantee the relatively complete recourse property. For our problem, this can be stated as follows: for every agent, (dropping the dependence on $i$ ), for all $\xi \in \Xi$, there exists $\left(\tilde{x}^{0}, \tilde{y}, \tilde{x}_{\xi}^{1}\right) \in X^{0} \times \mathbb{R}_{+}^{m} \times X_{\xi}^{1}$ such that

$$
\begin{aligned}
e_{l}^{0}-\tilde{x}_{l}^{0}-\left(T^{0} \tilde{y}\right)_{l} & \geq 0, \quad l=1, \ldots, L \\
e_{l, \xi}^{1}-\tilde{x}_{l, \xi}^{1}+\left(T_{\xi}^{1} \tilde{y}\right)_{l} & \geq 0, \quad l=1, \ldots, L, \forall \xi \in \Xi
\end{aligned}
$$

From the economic perspective, this assumption is weaker than the usual survability conditions, and can be interpreted as every agent being able to survive or participate in the economy, independently of the market prices. 


\subsection{Solving the agent's (stochastic) problem}

There are many alternatives methods to solve stochastic programs with recourse, but in this setup the use of the Progressive Hedging algorithm [19, 24] seems to have many advantages, in particular because solutions of the individual scenario subproblems are so readily available, cf. $\S 4$ with the Cobb-Douglas case.

The approach is based on relaxing, at the outset, the non-anticipativity constraint, namely that $x^{0}$ and $y$ aren't allowed to depend on $\xi$, and then, progressively enforcing this requirement. For now let's just limit ourselves to a description of the steps of the algorithm as it applies to the stochastic version of the (two-stage) agent's problem in the Cobb-Douglas case, generated by the transfer first approach described in $\S 4$.

Step 0. Set $\nu=0$. Pick $\rho>0, \bar{y}^{0}=0, w_{i}^{\nu}: \Xi \rightarrow \mathbb{R}^{m}$ such that $E_{i}\left\{w_{i}^{\nu}(\boldsymbol{\xi})\right\}=0$.

Step 1. For all $\xi \in \Xi$, let

$$
y_{i}^{\nu+1}(\xi) \in \operatorname{argmax}_{y}\left\{r_{i}^{\nu}(\xi, y)-\left\langle w_{i}^{\nu}, y\right\rangle-\frac{\rho}{2}\left|y-\bar{y}^{\nu}\right|^{2} \mid T_{i}^{0} y \leq e_{i}^{0}, \quad y \in \mathbb{R}_{+}^{m}\right\},
$$

where

$$
\begin{aligned}
r_{i}^{\nu}(\xi, y)= & \alpha_{i}^{0}\left(p^{0}\right)\left(\sum_{l=1}^{n} p_{l}^{0}\left(e_{i, l}^{0}-\left\langle T_{i, l}^{0}, y\right\rangle\right)\right) \\
& +\alpha_{i}^{1}\left(\xi, p^{1}(\xi)\right)\left(\sum_{l=1}^{n} p_{l}^{1}(\xi)\left(e_{i, l}^{1}(\xi)+\left\langle T_{i, l}^{1}(\xi), y\right\rangle\right)\right)
\end{aligned}
$$

and

$$
\alpha_{i}^{0}\left(p^{1}\right)=\prod_{j=1}^{n}\left(\frac{\beta_{i, j}^{0}}{p_{j}^{0}}\right)^{\beta_{i, j}^{0}}, \quad \alpha_{i}^{1}\left(\xi, p^{1}(\xi)\right)=\prod_{j=1}^{n}\left(\frac{\beta_{i, j}^{1}}{p_{j}^{1}(\xi)}\right)^{\beta_{i, j}^{1}} .
$$

Step 2. If $\xi \mapsto y_{i}^{\nu}(\xi)$ is a constant function, stop. $y^{\nu}(\xi)$, for any $\xi$, of course, determines the optimal activity levels and the corresponding vector and function $\left[x_{i}^{0},\left(x_{i}^{1}(\xi), \xi \in \Xi\right)\right]$ determine the optimal consumption plans. Otherwise, set $\bar{y}_{i}^{\nu+1}=E\left\{y_{i}^{\nu+1}(\boldsymbol{\xi})\right\}$,

$$
w_{i}^{\nu+1}(\xi)=w_{i}^{\nu}+\rho\left(y_{i}^{\nu+1}(\xi)-\bar{y}_{i}^{\nu+1}\right)
$$

and return to Step 1 with $\nu=\nu+1$.

Note that the optimization problem in Step $\mathbf{1}$ is a quadratic program of a very simple nature since it's completely separable. After carrying out some elementary calculations, it can be written in the form:

$$
\max \left\{\sum_{j=1}^{m}\left(\bar{c}_{j}(\xi) y_{j}-\frac{\rho}{2} y_{j}^{2}\right) \mid T_{i}^{0} y \leq e_{i}^{0}, \quad y \in \mathbb{R}_{+}^{m}\right\} .
$$

One could rely on general quadratic procedures to solve this particular problem, but a much more efficient procedure could be designed to deal with a problem of this particular type.

One final remark about this model is that it can be easily extended to the CES utility functions case, using the same transfer first approach of maximizing $r$ function. In this situation, is easy 
to see that the only difference is the sustitution of the linear coefficientes $\alpha$ by the ones given by the CES parameters, $\theta$. Furthermore, one can solve the general agent problem, relaxing the dependence of $x^{0}$ and $y$ on $\xi$, and apply the enforcing procedure to progressively converge to a deterministic solution.

\subsection{Augmented Walrasian and approximating scheme.}

In this section, we set the foundations of the augmentation techniques applied to the Dynamic Stochastic Equilibrium Model. A description of equilibrium points as maxinf points of the corresponding Walrasian, as well as the approximation scheme based in tight lopsided convergence of augmented Walrasian is provided. Finally, a general description of the computational implementation of the algorithm and numerical examples are analized.

As in the previous section, consider the stochastic equilibrium model, where given a price system $p=\left(p^{0},\left(p_{\xi}^{1}\right)_{\xi \in \Xi}\right)$, each agent $i$ solves

$$
\begin{aligned}
\max _{x^{0}, y, x^{1}} & u_{i}^{0}\left(x^{0}\right)+E_{i}\left\{u_{i}^{1}\left(\boldsymbol{\xi}, x_{\boldsymbol{\xi}}^{1}\right)\right\} \\
\text { so that } & \left\langle p^{0}, x^{0}+T_{i}^{0} y\right\rangle \leq\left\langle p^{0}, e_{i}^{0}\right\rangle, \\
& \left\langle p_{\xi}^{1}, x_{\xi}^{1}\right\rangle \leq\left\langle p_{\xi}^{1}, e_{i, \xi}^{1}+T_{i, \xi}^{1} y\right\rangle, \quad \forall \xi \in \Xi \\
& y \in \mathbb{R}_{+}^{m}, \quad x^{0} \in X_{i}^{0}, \quad x_{\xi}^{1} \in X_{i . \xi}^{1}, \quad \forall \xi \in \Xi,
\end{aligned}
$$

which defines the individual demand function $x_{i}(p)=\left(x_{i}^{0}(p),\left(x_{i, \xi}^{1}(p)\right)_{\xi \in \Xi}\right)$ and the individual transfer vector $y_{i}(p)$. Additionally, the excess supply function for this economy $s(p)=$

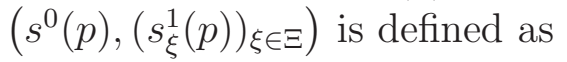

$$
\begin{aligned}
& s^{0}(p)=\sum_{i \in \mathcal{I}} e_{i}^{0}-x_{i}^{0}(p)-T_{i}^{0} y_{i}(p) \\
& s_{\xi}^{1}(p)=\sum_{i \in \mathcal{I}} e_{i, \xi}^{1}-x_{i, \xi}^{1}(p)+T_{i, \xi}^{1} y_{i}(p), \quad \forall \xi \in \Xi
\end{aligned}
$$

The Walrasian for this model is the function $W:\left(\Delta \times \Delta^{|\Xi|}\right) \times\left(\Delta \times \Delta^{|\Xi|}\right) \rightarrow \mathbb{R}$ defined by

$$
W(p, q)=\left\langle q^{0}, s^{0}(p)\right\rangle+\sum_{\xi \in \Xi}\left\langle q_{\xi}^{1}, s_{\xi}^{1}(p)\right\rangle
$$

A price system $\bar{p}=\left(\bar{p}^{0},\left(\bar{p}_{\xi}^{1}\right)_{\xi \in \Xi}\right)$ is an equilibrium price if $s(\bar{p}) \geq 0$, i.e., $s^{0}(\bar{p}) \geq 0$ and $s_{\xi}^{1}(p) \geq 0$, for every possible state $\xi \in \Xi$. Then, a equilibrium point for the dynamic stochastic model can be described as a maxinf point of the Walrasian. Again, the existence is granted by noting that $W$ turns out to be a Ky Fan function.

5.1 Theorem (stochastic equilibrium prices and maxinf-points). Consider the Walrasian function $W$ for the previous economy. Then, under local nonstatiation of preferences, every maxinfpoint $\bar{p}=\left(\bar{p}^{0},\left(\bar{p}_{\xi}^{1}\right)_{\xi \in \Xi}\right)$ of $W$ is an equilibrium point, i.e., $s^{0}(\bar{p}) \geq 0$ and $s_{\xi}^{1}(p) \geq 0$, for every possible state $\xi \in \Xi$. 
Proof. Considering that for every price system $p=\left(p^{0},\left(p_{\xi}^{1}\right)_{\xi \in \Xi}\right)$, under local nonsatiation preferences, the excess supply satisfies the Walras' law for the first stage and for every possible state of the second stage, i.e., $\left\langle p^{0}, s^{0}(p)\right\rangle=0$ and for every $\xi,\left\langle p_{\xi}^{1}, s_{x}^{1} i(p)\right\rangle=0$. Thus, for $\bar{p}$ a maxinf point of $W, W(\bar{p}, \cdot) \geq 0$. Considering $\left.q=\left(e^{j},\left(\bar{p}_{\xi}^{1}\right)_{\xi \in \Xi}\right), 0 \leq\langle q, s(\bar{p})\rangle=\left\langle e^{j}, s^{0}(\bar{p})\right\rangle+\sum_{\xi \in \Xi}\left\langle\bar{p}_{\xi}^{1}\right), s_{\xi}^{1}(\bar{p})\right\rangle$, which implies that $\left(s^{0}(\bar{p})\right)_{j} \geq 0$ for all $j$. For the second stage, given an scenario $\xi_{0} \in \Xi$, it suffices to take $q=\left(\bar{p}^{0},\left(\bar{p}_{1}^{1}, \ldots, \bar{p}_{\xi_{0}-1}^{1}, e^{j}, \bar{p}_{\xi_{0}+1}^{1}, \ldots, \bar{p}_{\Xi}^{1}\right)\right.$ and conclude by the same argument that $\left(s_{\xi_{0}}^{1}(\bar{p})\right)_{j} \geq 0$, for all $j$ and all $\xi_{0}$.

For the problem of finding equilibrium points, we will follow the approximating technique described in $\S 3$, where for a given Walrasian function $W$ for the stochastic economy, we consider the augmenting function $\sigma$, and an increasing sequence of positive scalars $r^{\nu} \nearrow \infty$, for which we defined the family of augmented Walrasian bifunctions $W^{\nu}$ as follows

$$
W^{\nu}(p, q)=\inf _{z \in \Delta \times \Delta \Xi}\left\{W(p, z)+r^{\nu} * \sigma^{*}(q-z)\right\},
$$

and the algorithmic procedure relies in the idea of finding approximating maxinf-points of this augmented Walrasian bifunctions for $\nu$ large enough. Finally, the following convergence result will guarantee the approximation to an equilibrium point for the initial economy.

5.2 Theorem (convergence of dynamic stochastic $\varepsilon$-maxinf points). Suppose that $p \mapsto s(p)$ is usc on $\Delta$. Consider the non-negative sequences $\left\{r^{\nu}: \nu \in \mathbb{N}\right\}$ and $\left\{\varepsilon^{\nu}: \nu \in \mathbb{N}\right\}$ such that $r^{\nu} \nearrow \infty, \varepsilon^{\nu} \searrow \varepsilon$, for $\varepsilon \geq 0$. Let $\left\{W^{\nu}: \nu \in \mathbb{N}\right\}$ be a family of Augmented Walrasian functions associated wich each augmenting parameter $r^{\nu}$. Let $p^{\nu} \in \varepsilon^{\nu}$-argmaxinf $W^{\nu}$ and $\bar{p}$ be a cluster point of $\left\{p^{\nu}: \nu \in \mathbb{N}\right\}$. Then $\bar{p} \in \varepsilon$-argmaxinf $W$. In particular, for $\varepsilon=0, \bar{p}$ is an equilibrium point.

Proof. The proof follows from the application of the Theorem 3.4, as it was used in the convergences results of sections $\S 3$ and $\S 4$ (Theorem 3.5, Theorem 4.2). Finally, the tight lopsided convergence of the sequence $\left\{W^{\nu}: \nu \in \mathbb{N}\right\}$ follows from the same argument.

\subsection{Numerical implementation and examples.}

Computationally, we proceed with a primal-dual iteration scheme as described in $\S 3$. Especial features for this type of economy are considered. In terms of the agent's problem, we can adopt a strategy solving the problem directly or solving it through the maximization of the overall reward function $r$.

On the other hand, the agent's problem is a stochastic program with relatively complete recourse, for which Progressive Hedging algorithm is implemented. Exploiting the structure of the agent's problem given by the separability in terms of the different scenarios in the second stage, combined with the progressive hedging approach, we provide two strategies, one sequential and another one parallel. The efficiency of these strategies will be discussed later and will basically depend on the size of the economy considered as the total amount of goods available.

Finally, the global strategy of solution adopted can be summarized in the following scheme: 
Step 0. Set $\nu=0$. Pick an initial price $p^{(0)}$ (for example, the centroid of the $\mathbb{R}^{G}$-simplex for each $\xi \in \Xi)$, and an augmenting parameter $r^{0}>0$. Define an strategy for the agent's problem, directly maximizing the utility function $u\left(x^{0}, y,\left(x_{\xi}^{1}\right)_{\xi \in \Xi}\right)$, or indirectly maximizing the overall reward function $r(y)$. Additionally, defined the procedure for the Progressive Hedging algorithm implementation, sequential or parallel.

Step 1. For all $i \in \mathcal{I}$, compute $x_{i}^{\nu}\left(p^{\nu}\right)$ applying Progresive Hedging algorithm to the agent's problem with the proper choice of strategies. With this, compute $s^{\nu}\left(p^{\nu}\right)$, and solve the Phase I iteration for the primal-dual scheme:

$$
q^{\nu+1} \in \operatorname{argmax}_{q}\left\{W_{r^{\nu}}\left(p^{\nu}, q\right) \mid q \in \Delta \times \Delta^{\Xi}\right\}
$$

which is a linear problem.

Step 2. Solve the Phase II, given by

$$
p^{\nu+1} \in \operatorname{argmax}_{p}\left\{W_{r^{\nu}}\left(p, q^{\nu+1}\right) \mid p \in \Delta \times \Delta^{\Xi}\right\} .
$$

Finally, check the optimality condition: if $\min s\left(p^{\nu+1}\right) \geq-\varepsilon$, stop. Otherwise, set $r^{\nu+1}>r^{\nu}$ and return to Step 1 with $\nu=\nu+1$.

\subsection{Numerical experimentation.}

5.3 Example (main example). The main example testing the numerical implementation of the augmented Walrasian algorithm is described for an economy consisting of seven goods: skilled job, unskilled job, leisure, consumption, risk free bond, and two stocks. We considered an economy with five agents, with utility functions of CES type, and nine posible scenarios in the second stage. On the other hand, the transformation matrices are the same for every agent at the first stage given by $T^{0}=\mathbf{I}$ and for the second stage are given by $T_{i, \xi}^{1}=\operatorname{diag}\left(d_{i, \xi}\right)$ for each agent $i=1, \ldots \mathcal{I}$, with

$$
\begin{aligned}
& d_{1, \xi}=\left(0,0,1+3 r / 4,0.7,1+r, R_{\xi}^{1}, R_{\xi}^{2}\right), \\
& d_{2, \xi}=\left(0,0,1+r / 2,0.8,1+r, R_{\xi}^{1}, R_{\xi}^{2}\right), \\
& d_{3, \xi}=\left(0,0,0,0.7,1+r, R_{\xi}^{1}, R_{\xi}^{2}\right), \\
& d_{4, \xi}=\left(0,0,1+r / 2,0.9,1+r, R_{\xi}^{1}, R_{\xi}^{2}\right), \\
& d_{5, \xi}=\left(0,0,1+r / 2,0.7,1+r, R_{\xi}^{1}, R_{\xi}^{2}\right) .
\end{aligned}
$$

where $r=3.25 \%$ and $R_{\xi}^{1}, R_{\xi}^{2}$ are given by the following table

\begin{tabular}{cc|ccc}
$\xi$ & & $\begin{array}{c}R_{(+)}^{2} \\
1.10\end{array}$ & $\begin{array}{c}R_{(=)}^{1} \\
1.00\end{array}$ & $\begin{array}{c}R_{(-)}^{1} \\
0.95\end{array}$ \\
\hline$R_{(+)}^{1}$ & 1.20 & 1 & 2 & 3 \\
$R_{(=)}^{1}$ & 1.00 & 4 & 5 & 6 \\
$R_{(-)}^{1}$ & 0.85 & 7 & 8 & 9
\end{tabular}


Agents' utility functions are CES type, with parameters can be found online ${ }^{2}$, as well as their initial endowments and survival sets. Additionally, we consider that every agent has the same beliefs over the scenarios on the second stage, given by $\pi_{i, \xi}=\frac{1}{9}, i \in \mathcal{I}, \xi \in \Xi$.

The algorithm is initialised with $p^{(0)}$ as the centroid of $\Delta \times \Delta^{\Xi}$, the augmenting function is $\sigma=\frac{1}{2}|\cdot|^{2}$, and the augmenting sequence of parameters $r^{\nu}$ is given by $r^{\nu}=1.259^{\nu}$. The trajectory of the prices $\left\{p^{\nu}\right\}$ for every iteration and the corresponding excess supply function $\left\{s\left(p^{\nu}\right)\right\}$ are described in figure 4. The algorithm was set for direct solution for the agent's problem and for Progressive Hedging, a sequential approach was considered. It finished after 62 iterations, with a total machine time of 28 [hrs].

Figure 4: Main example (Example 5.3), $\left\{p^{\nu}\right\}$ and $\left\{s\left(p^{\nu}\right)\right\}$

\section{Conclusions}

We introduced a new optimization methodology that allows the computation of equilibrium demand and prices for different economies. This new approach combines several elements of variational analysis, such as the notion of lopsided convergence and augmented Lagrangian technique for non-concave optimization problems.

Following [12], we characterize equilibrium prices as maxinf points for the so-called Walrasian bifunction for an exchange economy. The novelty of our approach relies in the approximation of the Walrasian by augmented Walrasian. Then, the computation of equilibrium points follows from the convergence of the sequence of maxinf points for the approximated problems.

We use this methodology to solve, as a prelude, the classical Arrow-Debreu general equilibrium model and, then, two periods exchange economies with uncertainty. For both models we got convergence in every numerical example, including a large scale problem in the stochastic case. A robust performance of the algorithm is always obtained, and it can be interpreted as a direct result of the augmentation procedure. One can appreciate stability of the iterations: by about half of the total iterations required to get a high tolerance-level solution. Furthermore, different numerical scenarios were tested, varying the augmenting function $\sigma$ and the augmenting parameter $r$. The results observed in these variations were not considered significantly different. The most efficient variant relied on the self-dual augmenting function with exponential growth in the augmenting parameter. Finally, for the stochastic problem, we tested an implementation of the algorithm based on a parallel computation for the agent problem.

The usage of the augmented Walrasian approximation for the computation of equilibrium points can be extended for more sophisticated economic models, as the one presented in [11], where financial markets, collateral, and retention goods are considered. Additionally, considering the structure of the problems, computational strategies that consider an efficient use of a parallel algorithm should improve the overall time performance.

\footnotetext{
${ }^{2}$ http://www.math.ucdavis.edu/ jderide/AugWal/AugWal.html
} 


\section{Acknowledgement}

This material is based upon work by Julio Deride and Roger Wets supported in part by the U.S. Army Research Laboratory and the U.S. Army Research Office under grant numbers W911NF10-1-0246 and W911NF-12-1-0273. We thank David Woodruff, University of California Davis, for his assistance with Pyomo, the optimization modeling language used in the computational implementation of our algorithm.

\section{References}

[1] K. Arrow and G. Debreu. Existence of an equilibrium for a competitive economy. Econometrica, 22:265-290, 1954.

[2] J.P. Aubin and I. Ekeland. Applied Nonlinear Analysis. Dover Publications, 2006.

[3] Adib Bagh. Augmenting the Walrasian. oral presentation, U. California, Davis, 2002.

[4] J. Birge and F. Louveaux. Introduction to Stochastic Programming. Springer Series in Operations Research and Financial Engineering. Springer, 2011.

[5] D. Brown, P. Demarzo, and C.Eaves. Computing equilibria when asset markets are incomplete. Econometrica, 64(1):1-27, 1996.

[6] D. Brown and F. Kubler. Computational Aspects of General Equilibrium Theory: Refutable Theories of Value. Springer Berlin Heidelberg, 2008.

[7] G. Debreu. Theory of Value. J. Wiley \& Sons, 1959.

[8] C. Eaves, editor. Homotopy Methods and Global Convergence. Springer, 2011.

[9] Inc. Gurobi Optimization. Gurobi optimizer reference manual, 2014.

[10] W.E. Hart, C. Laird, J.-P. Watson, and D.L. Woodruff. Pyomo - Optimization Modeling in Python. Springer Optimization and Its Applications. Springer, 2012.

[11] A. Jofré, R.T. Rockafellar, and R. Wets. General economic equilibrium with financial markets and retainability. Technical report, CMM-Universidad de Chile, 2014 (submitted).

[12] A. Jofré and R. Wets. Continuity properties of Walras equilibrium points. Annals of Operations Research, 114:229-243, 2002.

[13] A. Jofré and R. Wets. Variational convergence of bivariate functions: Lopsided convergence. Mathemathical Programming B, 116:275-295, 2009.

[14] A. Jofré and R. Wets. Variational convergence of bifunctions: motivating applications. SIAM J. on Optimization, 2014 (forthcoming).

[15] K.L. Judd. Numerical Methods in Economics. MIT Press, 1998. 
[16] A. Kirman. Elements of General Equilibrium Analysis. Wiley, 1998.

[17] M. Magill and M. Quinzii. Theory of Incomplete Markets. Number 0262632543 in MIT Press Books. The MIT Press, 2002.

[18] M. Powell. The BOBYQA algorithm for bound constrained optimization without derivatives. Cambridge NA Report NA2009/06, University of Cambridge, Cambridge, 2009.

[19] R.T. Rockafellar and R. Wets. Scenarios and policy aggregation in optimization under uncertainty. Mathematics of Operations Research, 16:119-147, 1991.

[20] R.T. Rockafellar and R. Wets. Variational Analysis, volume 317 of Grundlehren der Mathematischen Wissenschafte. Springer (3rd printing 2009), 1998.

[21] R. Saigal. A homotopy for solving large, sparse and structured fixed point problems. Mathematics of Operations Research, 8:557-578, 1983.

[22] H. Scarf and T. Hansen. The Computation of Economic Equilibria. Yale University Press, 1973.

[23] A. Wächter and L. Biegler. On the implementation of an interior-point filter line-search algorithm for large-scale nonlinear programming. Mathematical programming, 106:25-57, 2006 .

[24] R. Wets. The aggregation principle in scenario analysis and stochastic optimization. In S. Wallace, editor, Algorithms and Model Formulations in Mathematical Programming, NATO ASI Vol.51, pages 91-113. Springer-Verlag, NATO ASI Vol.51,, 1989. 